\title{
Boundary value problems for linear operators in ordered Banach spaces
}

by

\section{Gerd Herzog and Christoph Schmoeger (Karlsruhe)}

\begin{abstract}
We study boundary value problems of the type $A x=r, \varphi(x)=\varphi(b)$ $\left(\varphi \in M \subseteq E^{*}\right)$ in ordered Banach spaces.
\end{abstract}

1. Introduction. Let $E$ be a real ordered Banach space, $A: E \rightarrow E$ a continuous linear operator and $M$ a subset of $E^{*}$, the topological dual of $E$. We study Dirichlet type boundary value problems of the form

$$
\left\{\begin{array}{l}
A x=r \\
\varphi(x)=\varphi(b) \quad(\varphi \in M)
\end{array}\right.
$$

and we prove that under suitable assumptions on $A$ there exists a natural choice of $M$ such that this problem is uniquely solvable, and such that the solution $x$ depends monotonically on $r$ and $b$. Problems of this type emerge for example in discretization of linear elliptic boundary value problems [4, Chapter 4]. In this case the underlying space $E$ is finite-dimensional and is ordered coordinatewise, in general.

To illustrate our results we consider the following example. Let $E=$ $C([0,1], \mathbb{R})$ be endowed with the pointwise ordering, let $B_{n}$ denote the Bernstein operator

$$
\left(B_{n} f\right)(x)=\sum_{k=0}^{n} f\left(\frac{k}{n}\right)\left(\begin{array}{l}
n \\
k
\end{array}\right) x^{k}(1-x)^{n-k},
$$

and let $m \in \mathbb{N}$. We will see that the problem

$$
\left\{\begin{array}{l}
\left(B_{n}-\mathrm{id}\right)^{m} f=g, \\
f(0)=\alpha, \quad f(1)=\beta
\end{array}\right.
$$

is uniquely solvable for each $g$ in the range of $B_{n}-$ id and each $\alpha, \beta \in \mathbb{R}$, and that the solution depends monotonically on $\alpha, \beta$ and $g$.

2010 Mathematics Subject Classification: Primary 47B60; Secondary 47A50.

Key words and phrases: ordered Banach spaces, quasimonotone increasing operators, Fredholm operators, boundary value problems. 
2. Main result. Let $E$ be a real Banach space ordered by a cone $K$. A cone $K$ is a nonempty closed convex subset of $E$ such that $\lambda K \subseteq K$ $(\lambda \geq 0)$, and $K \cap(-K)=\{0\}$. As usual $x \leq y: \Leftrightarrow y-x \in K$. For $x \leq y$ let $[x, y]$ denote the order interval of all $z$ with $x \leq z \leq y$. We assume in the following that $K$ is normal (that is, $0 \leq x \leq y \Rightarrow\|x\| \leq \gamma\|y\|$ for some constant $\gamma \geq 1$ ), and has nonempty interior int $K$. Let $K^{*}$ denote the dual cone of $K$, that is, the set of all $\varphi \in E^{*}$ with $\varphi(x) \geq 0(x \geq 0)$. Let $L(E)$ denote the Banach algebra of all continuous linear operators $A: E \rightarrow E$, and for $A \in L(E)$ let $A^{*}$ denote its adjoint.

An operator $A \in L(E)$ is called quasimonotone increasing [11] if

$$
x \in K, \varphi \in K^{*}, \varphi(x)=0 \Rightarrow \varphi(A x) \geq 0 .
$$

It is well known [8] that $A \in L(E)$ is quasimonotone increasing if and only if $\exp (t A)(K) \subseteq K$ (that is, $\exp (t A)$ is a positive operator) for each $t \geq 0$. In this case also $\exp \left(t A^{*}\right)\left(K^{*}\right) \subseteq K^{*}(t \geq 0)$.

Next, if any $p \in \operatorname{int} K$ is fixed, then we may renorm $E$ equivalently by the Minkowski functional of the order interval $[-p, p]$. This norm $\|\cdot\|$ satisfies

$$
-c p \leq x \leq c p \Leftrightarrow\|x\| \leq c .
$$

Under these settings we have

$$
\|\varphi\|=\varphi(p) \quad\left(\varphi \in K^{*}\right)
$$

and we set

$$
C^{*}:=\left\{\varphi \in K^{*}: \varphi(p)=1\right\}=\left\{\varphi \in E^{*}: \varphi(p)=\|\varphi\|=1\right\} .
$$

Moreover, we define a continuous sublinear functional $S: E \rightarrow \mathbb{R}$ by

$$
S(x)=\min \{\lambda \in \mathbb{R}: x \leq \lambda p\} .
$$

Note [6] that $S$ is increasing with respect to the order given by $K$, that

$$
S(x)=\max \left\{\varphi(x): \varphi \in C^{*}\right\},
$$

and that

$$
\|x\|=\max \{S(-x), S(x)\} \quad(x \in E) .
$$

We denote by $N(\cdot), R(\cdot)$, and $\operatorname{ext}(\cdot)$ the kernel and range of a linear operator, and the set of all extremal points of a subset of a Banach space, respectively.

The aim of this paper is to prove the following results. Let $A \in L(E)$ be quasimonotone increasing with

$$
N(A) \cap \operatorname{int} K \neq \emptyset
$$

and fix $p \in N(A) \cap$ int $K$. Let $E$ be normed with respect to this $p$. Moreover 
we assume that $t \mapsto \exp (t A)$ is strongly Cesàro integrable, that is,

$$
\frac{1}{t} \int_{0}^{t} \exp (\tau A) x d \tau
$$

is convergent in $E$ as $t \rightarrow \infty$ for each $x \in E$.

LEMMA 1. Under the assumptions above we have: For each $x_{0} \in E$ with $A x_{0} \geq 0$ there exists a unique $y_{0} \in N(A)$ such that

$$
x_{0} \leq y_{0}, \quad \varphi\left(x_{0}\right)=\varphi\left(y_{0}\right) \quad\left(\varphi \in \operatorname{ext}\left(N\left(A^{*}\right) \cap C^{*}\right)\right),
$$

and

$$
S\left(x_{0}\right)=\max \left\{\varphi\left(x_{0}\right): \varphi \in \operatorname{ext}\left(N\left(A^{*}\right) \cap C^{*}\right)\right\} .
$$

Theorem 1. Let $A$ be as in Lemma 1. Then for each $m \in \mathbb{N}$ the Dirichlet type boundary value problem

$$
\left\{\begin{array}{l}
A^{m} x=r \\
\varphi(x)=\varphi(b) \quad\left(\varphi \in \operatorname{ext}\left(N\left(A^{*}\right) \cap C^{*}\right)\right)
\end{array}\right.
$$

is uniquely solvable in $E$ for each $r \in R\left(A^{m}\right)$ and $b \in E$, and the solution depends increasingly on $b$, decreasingly on $r$ if $m$ is odd, and increasingly on $r$ if $m$ is even. If in addition $R\left(A^{m}\right)$ is closed, then there exists a constant c such that

$$
\|x\| \leq c\|r\|+\|b\| \quad\left(r \in R\left(A^{m}\right), b \in E\right) .
$$

Remark. Part (3) of Lemma 1 and Theorem 1 for $m=1$ can be considered in analogy to the classical maximum principle and to the solution behaviour of linear second order BVPs [10, or corresponding BVPs for difference equations [4, Section 4.4].

3. Preliminaries. We make use of the following lemmata. We assume that $p \in \operatorname{int} K$, and that $E$ is normed by the Minkowski functional of the order interval $[-p, p]$. We denote by $m_{+}[x, y]$ the right hand side directional derivative [9, Lemma II.5.6]:

$$
m_{+}[x, y]=\lim _{h \rightarrow 0+} \frac{\|x+h y\|-\|x\|}{h} .
$$

Lemma 2. Let $A \in L(E)$ be quasimonotone increasing. Then

$$
\|\exp (t A) x\| \leq \exp \left(t m_{+}[p, A p]\right)\|x\| \quad(x \in E, t \geq 0) .
$$

For the proof of Lemma 2 see [5].

Lemma 3. Let $A \in L(E)$ be quasimonotone increasing with $A p=0$. Let $x \in E$ and $u(t)=\exp (t A) x(t \geq 0)$. Then

1. $\|u(t)\| \leq\|x\|(t \geq 0)$,

2. $t \mapsto S(u(t))$ is decreasing on $[0, \infty)$, 
3. $A x \geq 0 \Rightarrow S(u(t))=S(x)(t \geq 0)$.

Proof. 1. This follows by Lemma 2.

2. We have $x \leq S(x) p$, thus

$$
u(t)=\exp (t A) x \leq S(x) \exp (t A) p=S(x) p \quad(t \geq 0) .
$$

Therefore $S(u(t)) \leq S(x)(t \geq 0)$. Hence, if $0 \leq t_{1} \leq t_{2}$ we obtain

$$
S\left(u\left(t_{2}\right)\right)=S\left(\exp \left(\left(t_{2}-t_{1}\right) A\right) \exp \left(t_{1} A\right) x\right) \leq S\left(\exp \left(t_{1} A\right) x\right)=S\left(u\left(t_{1}\right)\right) .
$$

3 . If $A x \geq 0$, then $u$ is increasing on $[0, \infty)$ (since $u^{\prime}(t)=\exp (t A) A x \geq 0$ in this case). Since $S$ is increasing on $E$ it follows that $t \mapsto S(u(t))$ is monotone increasing and (by item 2) $t \mapsto S(u(t))$ is monotone decreasing.

4. Proof of Lemma 1 and Theorem 1. For $x \in E$ we set

$$
Q x:=\lim _{t \rightarrow \infty} \frac{1}{t} \int_{0}^{t} \exp (\tau A) x d \tau \quad(x \in E) .
$$

Then $Q \in L(E)$, and note that

$$
\left(Q^{*} \varphi\right)(x)=\varphi(Q x)=\lim _{t \rightarrow \infty} \frac{1}{t} \int_{0}^{t} \varphi(\exp (\tau A) x) d \tau
$$

for each $x \in E$ and $\varphi \in E^{*}$.

By means of Lemma 2 we find that $\|\exp (t A)\|=1(t \geq 0)$. Therefore

$$
\begin{aligned}
Q A x & =A Q x=\lim _{t \rightarrow \infty} \frac{1}{t} \int_{0}^{t} A \exp (\tau A) x d \tau \\
& =\lim _{t \rightarrow \infty} \frac{1}{t}(\exp (t A) x-x)=0 \quad(x \in E) .
\end{aligned}
$$

Thus $Q(E) \subseteq N(A)$, and since $Q(x)=x(x \in N(A))$ we see that $Q$ is a projection onto $N(A)$. From the definition of $Q$ we immediately get

$$
Q(K) \subseteq K, \quad\|Q\|=1 .
$$

Thus

$$
Q^{*}\left(K^{*}\right) \subseteq K^{*}, \quad\left\|Q^{*}\right\|=1 .
$$

Moreover $Q^{*}$ is a projection onto $N\left(A^{*}\right)$. Indeed, if $\varphi=Q^{*} \psi$, then

$$
\left(A^{*} \varphi\right)(x)=\left(A^{*} Q^{*} \psi\right)(x)=\psi(Q A x)=0 \quad(x \in E),
$$

thus $Q^{*}\left(E^{*}\right) \subseteq N\left(A^{*}\right)$, and if $\varphi \in N\left(A^{*}\right)$, then

$$
\left(Q^{*} \varphi\right)(x)=\lim _{t \rightarrow \infty} \frac{1}{t} \int_{0}^{t} \varphi(\exp (\tau A) x) d \tau=\lim _{t \rightarrow \infty} \frac{1}{t} \int_{0}^{t} \varphi(x) d \tau=\varphi(x) \quad(x \in E),
$$

therefore $Q^{*} \varphi=\varphi$. 
We set $u(t)=\exp (t A) x_{0}(t \geq 0), g(0)=x_{0}$ and

$$
g(t)=\frac{1}{t} \int_{0}^{t} u(\tau) d \tau \quad(t>0) .
$$

Then $g$ is continuous and increasing on $[0, \infty)$ (since $u$ is increasing), and therefore $y_{0}:=Q x_{0} \geq x_{0}$. Moreover, by Lemma 3 , we know that $S(u(t))=$ $S\left(x_{0}\right)(t \geq 0)$. Since $S$ is sublinear we have

$$
S(g(t)) \leq \frac{1}{t} \int_{0}^{t} S(u(\tau)) d \tau=S\left(x_{0}\right) \quad(t>0) .
$$

Thus $S\left(y_{0}\right) \leq S\left(x_{0}\right)$. Since $S$ is increasing, in addition $S\left(x_{0}\right) \leq S\left(y_{0}\right)$, and so $S\left(x_{0}\right)=S\left(y_{0}\right)$. Since

$$
\varphi\left(y_{0}\right)=\varphi\left(Q x_{0}\right)=\left(Q^{*} \varphi\right)\left(x_{0}\right)=\varphi\left(x_{0}\right) \quad\left(\varphi \in N\left(A^{*}\right)\right)
$$

we have $y_{0} \in N(A)$ satisfying (2). Uniqueness of $y_{0}$ will follow from unique solvability of (4) (with $m=1, r=0$ and $b=x_{0}$ ).

Next, we choose $\psi_{0} \in C^{*}$ such that $\psi_{0}\left(x_{0}\right)=S\left(x_{0}\right)$, and we set $\varphi_{0}:=$ $Q^{*} \psi_{0}$. Then $\varphi_{0} \in N\left(A^{*}\right) \cap K^{*}$, and

$$
\varphi_{0}(p)=\left(Q^{*} \psi_{0}\right)(p)=\psi_{0}(Q p)=\psi_{0}(p)=1,
$$

thus $\varphi_{0} \in N\left(A^{*}\right) \cap C^{*}$.

We set $v(t)=\exp \left(t A^{*}\right) \psi_{0}(t \geq 0), h(0)=\psi_{0}$ and

$$
h(t)=\frac{1}{t} \int_{0}^{t} v(\tau) d \tau \quad(t>0) .
$$

Now $h$ is continuous, and with $u$ also $\psi_{0} \circ u$ and $(h(\cdot))\left(x_{0}\right)$ are increasing on $[0, \infty)$. For $t>0$ we have

$$
\begin{aligned}
(h(t))\left(x_{0}\right) & =\frac{1}{t} \int_{0}^{t}\left(\exp \left(\tau A^{*}\right) \psi_{0}\right)\left(x_{0}\right) d \tau \\
& =\frac{1}{t} \int_{0}^{t} \psi_{0}(u(\tau)) d \tau \rightarrow \psi_{0}\left(Q x_{0}\right)=\varphi_{0}\left(x_{0}\right) \quad(t \rightarrow \infty) .
\end{aligned}
$$

Thus $\psi_{0}\left(x_{0}\right)=(h(0))\left(x_{0}\right) \leq \varphi_{0}\left(x_{0}\right)$. Now

$$
S\left(x_{0}\right)=\psi_{0}\left(x_{0}\right) \leq \varphi_{0}\left(x_{0}\right) \leq S\left(x_{0}\right),
$$

and therefore $\varphi_{0}\left(x_{0}\right)=S\left(x_{0}\right)$. At this point we know that

$$
S\left(x_{0}\right)=\max \left\{\varphi\left(x_{0}\right): \varphi \in N\left(A^{*}\right) \cap C^{*}\right\} .
$$

Since $N\left(A^{*}\right) \cap C^{*}$ is a convex and weak-* compact subset of $E^{*}$, and since $\varphi \mapsto \varphi\left(x_{0}\right)$ is an affine function on $N\left(A^{*}\right) \cap C^{*}$, its maximum is attained at an extremal point (see [2, Prop. 7.9]). Thus we have (3). 
To prove that (4) has at most one solution first note that $N\left(A^{2}\right)=N(A)$. Indeed if $y \in N\left(A^{2}\right)$ then

$$
\|\exp (t A) y\|=\|y+t A y\| \leq\|y\| \quad(t \geq 0) .
$$

Thus $A y=0$. Consequently $N\left(A^{n}\right)=N(A)(n \in \mathbb{N})$. Now, consider a solution $x \in E$ of the homogeneous problem

$$
\left\{\begin{array}{l}
A^{m} x=0, \\
\varphi(x)=0 \quad\left(\varphi \in \operatorname{ext}\left(N\left(A^{*}\right) \cap C^{*}\right)\right) .
\end{array}\right.
$$

Then $x \in N\left(A^{m}\right)=N(A)$, and according to (3) we have $S(x)=S(-x)=0$. Thus $x=0$.

To prove the existence of a solution of (4) we consider

$$
B:(I-Q)(E) \rightarrow R\left(A^{m}\right)
$$

defined by $B x=A^{m} x(x \in(I-Q)(E))$. Then $B$ is bijective, and $A^{m} B^{-1} r=r$. Moreover

$$
\varphi(Q x)=\left(Q^{*} \varphi\right)(x)=\varphi(x) \quad\left(x \in E, \varphi \in N\left(A^{*}\right)\right) .
$$

Now,

$$
x=B^{-1} r+Q b
$$

satisfies $A^{m} x=r$, and for each $\varphi \in N\left(A^{*}\right)$ we have

$$
\varphi(x)=\varphi\left(B^{-1} r\right)+\varphi(Q b)=0+\varphi(b)=\varphi(b) .
$$

In particular $x$ is the solution of (4), and since $Q$ is a positive operator, we see that the solution of (4) depends increasingly on $b$.

Next, let $r_{1}, r_{2} \in R\left(A^{m}\right)$ with $r_{1} \leq r_{2}$, and let $x_{1}, x_{2}$ be the solutions of

$$
\left\{\begin{array}{l}
A^{m} x_{i}=r_{i}, \\
\varphi\left(x_{i}\right)=\varphi(b) \quad\left(\varphi \in \operatorname{ext}\left(N\left(A^{*}\right) \cap C^{*}\right)\right)
\end{array}\right.
$$

for $i=1,2$. Then $z=x_{2}-x_{1}$ is the solution of

$$
\left\{\begin{array}{l}
A^{m} z=r_{2}-r_{1} \geq 0 \\
\varphi(z)=0 \quad\left(\varphi \in \operatorname{ext}\left(N\left(A^{*}\right) \cap C^{*}\right)\right)
\end{array}\right.
$$

and

$$
\varphi\left(A^{m-1} z\right)=\left(\left(A^{*}\right)^{m-1} \varphi\right)(z)=0 \quad\left(\varphi \in \operatorname{ext}\left(N\left(A^{*}\right) \cap C^{*}\right)\right) .
$$

By means of (3) we have $S\left(A^{m-1} z\right)=0$, so $A^{m-1} z \leq 0$. Thus, $-z$ satisfies

$$
\left\{\begin{array}{l}
A^{m-1}(-z) \geq 0 \\
\varphi(-z)=0 \quad\left(\varphi \in \operatorname{ext}\left(N\left(A^{*}\right) \cap C^{*}\right)\right)
\end{array}\right.
$$

and repeating this step we obtain

$$
A^{m-2}(-z) \leq 0, \quad A^{m-3} z \leq 0, \quad \cdots, \quad(-1)^{m-1} z \leq 0,
$$

which means

$$
(-1)^{m} x_{1} \leq(-1)^{m} x_{2}
$$


To prove (5) we assume in addition that $R\left(A^{m}\right)$ is closed. Since $(I-Q)(E)$ is a closed subspace of $E$, and since $B:(I-Q)(E) \rightarrow R\left(A^{m}\right)$ is bijective, we have a continuous inverse $B^{-1}: R\left(A^{m}\right) \rightarrow(I-Q)(E)$ in this case. Set $c:=\left\|B^{-1}\right\|$. Now the solution $x=B^{-1} r+Q b$ of (4) satisfies

$$
\|x\| \leq\left\|B^{-1} r\right\|+\|Q b\| \leq c\|r\|+\|b\| \text {. }
$$

5. Fredholm operators. In special cases the convergence of the integral (1) for each $x \in E$ is automatically true under the remaining assumptions of Lemma 1. This is the case for example if $E$ is reflexive [3, VIII.7.3]. For general $E$ we can even prove a bit more if $A$ is a Fredholm operator.

An operator $A \in L(E)$ is called a Fredholm operator if

$$
\alpha(A):=\operatorname{dim} N(A)<\infty, \quad \beta(A):=\operatorname{codim} R(A)<\infty .
$$

In this case $R(A)$ is closed [7, Prop. 36.3], $A^{n}$ is a Fredholm operator $(n \in \mathbb{N})$ [7. Prop. 25.3], and $A^{*}$ is a Fredholm operator [7, Prop. 27.3]. Moreover $\operatorname{ind}(A):=\alpha(A)-\beta(A)$ is called the index of $A$.

For $A \in L(E)$ we define

$$
\begin{aligned}
& a(A):=\min \left\{n \geq 0: N\left(A^{n}\right)=N\left(A^{n+1}\right)\right\}, \\
& d(A):=\min \left\{n \geq 0: R\left(A^{n}\right)=R\left(A^{n+1}\right)\right\},
\end{aligned}
$$

with $\min \emptyset:=\infty$. Now, $A$ is called chain-finite if $a(A)<\infty$ and $d(A)<\infty$. In this case $a(A)=d(A)$ [7, Prop. 38.3]. We will use the following facts from the Riesz-Schauder theory of compact operators [7, Prop. 40.1]:

If $T \in L(E)$ is compact and $A=T-$ id, then $A$ and $A^{*}$ are Fredholm operators with

$$
\begin{aligned}
& \alpha(A)=\beta(A)=\alpha\left(A^{*}\right)=\beta\left(A^{*}\right), \\
& a(A)=d(A)=a\left(A^{*}\right)=d\left(A^{*}\right)<\infty .
\end{aligned}
$$

If $T \in L(E)$, and $T^{k}$ is compact for some $k \in \mathbb{N}$ (we call $T$ power compact in this case), $A=T-\mathrm{id}, A_{1}=T^{k}-\mathrm{id}$, and

$$
A_{2}=\mathrm{id}+T+\cdots+T^{k-1},
$$

then $A_{1}$ is a Fredholm operator of index $0, a\left(A_{1}\right)=d\left(A_{1}\right)<\infty$, and

$$
A_{1}=A A_{2}=A_{2} A \text {. }
$$

Therefore

$$
N(A) \subseteq N\left(A_{1}\right), \quad R\left(A_{1}\right) \subseteq R(A),
$$

and so

$$
\alpha(A) \leq \alpha\left(A_{1}\right)<\infty, \quad \beta(A) \leq \beta\left(A_{1}\right)<\infty .
$$

Thus $A$ is a Fredholm operator, and by [7, Exerc. 2, Sect. 38] we have

$$
\operatorname{ind}(A)=0, \quad a(A)=d(A)<\infty .
$$


The following results are stated for operators of the form $A=T$-id, but also hold for $A=T-\lambda$ id with $\lambda>0$. We assume without loss of generality that $\lambda=1$, since division by $\lambda$ does not affect the following considerations.

Theorem 2. Let $T \in L(E)$ be power compact, let $A=T$ - id be quasimonotone increasing, and let $N(A) \cap \operatorname{int} K \neq \emptyset$. Then (1) is convergent as $t \rightarrow \infty$ for each $x \in E$. Moreover $A$ is a Fredholm operator of index 0 and

$$
a(A)=d(A)=1 .
$$

By Theorems 1 and 2 we get the following result on problem (4).

Theorem 3. Let $T \in L(E)$ be power compact, let $A=T$ - id be quasimonotone increasing, let $N(A) \cap \operatorname{int} K \neq \emptyset$, and let $m \in \mathbb{N}$. Then problem (4) is uniquely solvable in $E$ for each $r \in R(A)$ and $b \in E$, and the solution depends increasingly on $b$, decreasingly on $r$ if $m$ is odd, and increasingly on $r$ if $m$ is even, and there exists a constant $c$ such that

$$
\|x\| \leq c\|r\|+\|b\| \quad(r \in R(A), b \in E) .
$$

Proof. By Theorem 2, $A$ is a Fredholm operator of index 0, hence $R\left(A^{m}\right)$ is closed. Since $d(A)=1$ we have $R(A)=R\left(A^{m}\right)$. The assertion now follows from Theorem 1.

REMARK. Theorem 3 applies to our introductory example. There $E=$ $C([0,1], \mathbb{R})$ is ordered by the cone

$$
K=\{f \in E: f(t) \geq 0(t \in[0,1])\} .
$$

The operator $B_{n}$ is compact, and since $B_{n}$ is increasing, $A=B_{n}-\mathrm{id}$ is quasimonotone increasing. The function $p(t)=1(t \in[0,1])$ is in $\operatorname{int} K$, and $A p=0$. The norm induced by $p$ is the maximum norm. Since $B_{n}^{k} \rightarrow B_{1}$ pointwise on $E$ as $k \rightarrow \infty$ (cf. [1]) we see that $N(A)=\{1, t\}$. Hence $\alpha\left(A^{*}\right)=$ $\alpha(A)=2$. Let $\varphi_{0}, \varphi_{1}$ denote the functionals $\varphi_{0}(f)=f(0), \varphi_{1}(f)=f(1)$ $(f \in E)$. Then $\varphi_{0}, \varphi_{1} \in N\left(A^{*}\right)$, and since $\alpha\left(A^{*}\right)=2$ we have $N\left(A^{*}\right)=$ $\operatorname{span}\left\{\varphi_{0}, \varphi_{1}\right\}$. Thus

$$
N\left(A^{*}\right) \cap C^{*}=\left\{\mu \varphi_{0}+(1-\mu) \varphi_{1}: \mu \in[0,1]\right\},
$$

and therefore

$$
\left.\operatorname{ext}\left(N\left(A^{*}\right) \cap C^{*}\right)\right)=\left\{\varphi_{0}, \varphi_{1}\right\} .
$$

6. Proof of Theorem 2. Fix $p \in N(A) \cap$ int $K$ and again let $E$ be normed with respect to this $p$. Let $x \in E$. We have

$$
\exp (t A) x=\exp (-t)\left(\sum_{j=0}^{k-1} \frac{t^{j} T^{j} x}{j !}+T^{k} \sum_{j=k}^{\infty} \frac{t^{j} T^{j-k} x}{j !}\right) .
$$


Since

$$
\frac{d^{k}}{d t^{k}}\left(\sum_{j=k}^{\infty} \frac{t^{j} T^{j-k} x}{j !}\right)=\exp (t T)
$$

we have

$$
h(t):=\exp (-t)\left(\sum_{j=k}^{\infty} \frac{t^{j} T^{j-k} x}{j !}\right)=\int_{0}^{t} \int_{0}^{t_{1}} \ldots \int_{0}^{t_{k-1}} \exp \left(t_{k} T-t \mathrm{id}\right) x d t_{k} \ldots d t_{2} d t_{1} .
$$

Since $t_{k} T-t$ id is quasimonotone increasing for $t_{k}, t \geq 0$, and since

$$
m_{+}\left[p,\left(t_{k} T-t \mathrm{id}\right) p\right]=m_{+}\left[p,\left(t_{k}-t\right) p\right]=t_{k}-t
$$

we deduce by Lemma 2 that

$$
\left\|\exp \left(t_{k} T-t \mathrm{id}\right) x\right\| \leq \exp \left(t_{k}-t\right)\|x\| \quad\left(t_{k}, t \geq 0\right)
$$

Thus

$$
\|h(t)\| \leq \int_{0}^{t} \int_{0}^{t_{1}} \ldots \int_{0}^{t_{k-1}} \exp \left(t_{k}-t\right)\|x\| d t_{k} \ldots d t_{2} d t_{1}
$$

and evaluation of this integral proves that $h$ is bounded on $[0, \infty)$. Now

$$
\begin{aligned}
& \frac{1}{t} \int_{0}^{t} \exp (\tau A) x d \tau \\
& \quad=\frac{1}{t} \int_{0}^{t} \exp (-\tau)\left(\sum_{j=0}^{k-1} \frac{\tau^{j} T^{j} x}{j !}\right) d \tau+T^{k}\left(\frac{1}{t} \int_{0}^{t} h(\tau) d \tau\right) \quad(t>0)
\end{aligned}
$$

proves that

$$
\left\{\frac{1}{t} \int_{0}^{t} \exp (\tau A) x d \tau: t>0\right\}
$$

is relatively compact, and according to [3, VIII.7.1],

$$
\lim _{t \rightarrow \infty} \frac{1}{t} \int_{0}^{t} \exp (\tau A) x d \tau
$$

exists.

Next, we have already seen that $A$ is a Fredholm operator with

$$
\operatorname{ind}(A)=0, \quad a(A)=d(A)<\infty,
$$

since $T$ is power compact. In the proof of Theorem 1 we have seen that $N\left(A^{2}\right)=N(A)$. Thus $a(A) \leq 1$, and since $A$ is not injective, $a(A)=1$. Thus $d(A)=1$ too. 


\section{References}

[1] U. Abel and M. Ivan, Over-iterates of Bernstein's operators: a short and elementary proof, Amer. Math. Monthly 116 (2009), 535-538.

[2] J. B. Conway, A Course in Functional Analysis, Grad. Texts in Math. 96, Springer, New York, 1985.

[3] N. Dunford and J. T. Schwartz, Linear Operators. I. General Theory, Pure Appl. Math. 7, Interscience, New York, 1958.

[4] W. Hackbusch, Elliptic Differential Equations: Theory and Numerical Treatment, Springer Ser. Comput. Math. 18, Springer, Berlin, 1992.

[5] G. Herzog, One-sided estimates for linear quasimonotone increasing operators, Numer. Funct. Anal. Optim. 19 (1998), 549-555.

[6] G. Herzog and R. Lemmert, One-sided estimates for quasimonotone increasing functions, Bull. Austral. Math. Soc. 67 (2003), 383-392.

[7] H. G. Heuser, Functional Analysis, Wiley, Chichester, 1982.

[8] R. Lemmert and P. Volkmann, On the positivity of semigroups of operators, Comment. Math. Univ. Carolin. 39 (1998), 483-489.

[9] R. H. Martin, Nonlinear Operators and Differential Equations in Banach Spaces, Krieger, Malabar, FL, 1987.

[10] M. H. Protter and H. F. Weinberger, Maximum Principles in Differential Equations, Prentice-Hall, Englewood Cliffs, NJ, 1967.

[11] P. Volkmann, Gewöhnliche Differentialungleichungen mit quasimonoton wachsenden Funktionen in topologischen Vektorräumen, Math. Z. 127 (1972), 157-164.

Gerd Herzog, Christoph Schmoeger

Institut für Analysis

Karlsruher Institut für Technologie

D-76128 Karlsruhe, Germany

E-mail: gerd.herzog2@kit.edu

christoph.schmoeger@kit.edu

Received September 22, 2009

Revised version January 20, 2010 